\title{
Addressing the gaps: therapeutic and prognostic considerations in temporomandibular joint osteoarthrosis
}

\begin{abstract}
Background: Osteoarthritis is a common chronic degenerative joint disease but its involvement in Temporomandibular joint (TMJ) is relatively uncommon. Various treatment modalities including conservative and surgical therapies are available.
\end{abstract}

Aim: To analyze different techniques of management to know whether the noninvasive conservative line of treatment is effective for the patients suffering from TMJ osteoarthritis.

Material and Method: This research included studies that examined the management of TMJ osteoarthritis. The data in this article are supported by a Medline and Google scholar search. These were augmented by references from these articles. Data from the articles were extracted using a pre-defined data-extraction form.

Result: The first line of treatment of was found to be conservative therapy. Conservative modality of treatment was effective and caused least morbidity. Irreversible oral rehabilitation was seen generally avoided in active stages of TMJ osteoarthritis.

Conclusion: After analyzing different techniques of management, it is obvious that the noninvasive conservative line of treatment is effective and should be the first line and cornerstone of therapy for the patients suffering from TMJ osteoarthritis.
Volume 8 Issue 6 - 2017

Mohan Bansal
Department ENT Head and Neck surgery GK Hospital, India

Correspondence: Gujarat Adani Institute of Medical Sciences Department ENT Head and Neck surgery GK Hospital Bhuj Gujarat 37000I, India, Tel +919726859077, Email mohanbansal@yahoo.com

Received: September 09, 2017 | Published: October 06, 2017

Keywords: osteoarthritis, temporomandibular joint, degenerative joint disease

Abbreviations: TMJ, temporomandibular joint; TMD, temporomandibular disorders; COX-2, cyclooxygenase-2; NSAIDs, non-steroidal anti-inflammatory drugs; HA, hyaluronic acid; MMPs, matrix metalloproteinase

\section{Introduction}

Temporomandibular disorders (TMD) consist of not only the internal derangement of Temporomandibular joint (TMJ) but also areas extrinsic to TMJ. ${ }^{1}$ Osteoarthritis can also affect Temporomandibular joint (TMJ) where it involves the cartilage, subchondral bone, and synovial membrane and results in TMJ remodeling and abrasion of articular cartilage. ${ }^{2,3}$ Epidemiologic findings of TMJ osteoarthritis are similar to generalized osteoarthritis. ${ }^{4}$ The clinical manifestations of TMJ osteoarthritis occurs in 8-16\% of population ${ }^{5,6}$ and involvement may be unilateral or bilateral. Females are affected more. The estrogen receptor $\alpha$-polymorphism can cause increased pain susceptibility in female patients.?

Osteoarthritis has multiple risk factors such as age, genetics, trauma, joint and muscle disorders, infection, generalized osteoarthritis, congenital and developmental abnormality and idiopathic degenerative process. Trauma includes past fractures, jaw trauma, repetitive high-impact and torsional loads. Ligament laxity, internal derangements and inadequate muscle strength are examples of joint and muscle disorders. ${ }^{8-10}$ The natural course of the disease consists of periods of remission. It is divided into three slow progressive phases. The entire disease process takes about 5.5years up to the final burnout phase. ${ }^{11}$

The most common clinical features are pain, restriction in mouth opening, and crepitus. The joint warmth is also absent. Pain is generally dull aching and common during the initial phases. Some patients have morning joint stiffness and increased sensitivity to cold and damp. The pain relieving factors include rest and NSAIDs. In later stages patient may develop facial skeletal remodeling. Patients have chin deviation and that occurs toward the affected side. Occlusal discrepancies like malocclusion are not uncommon. ${ }^{12}$ Occlusal changes include reduced overbite and anterior open bite. ${ }^{10,13}$ Internal derangements occur in about one-third of the patients. ${ }^{14}$

Various treatment modalities including conservative and surgical therapies are available. The aim of this study is to analyze different techniques of management to know whether the noninvasive conservative line of treatment is effective for the patients suffering from TMJ osteoarthritis.

\section{Material and methods}

This study was granted an exception from ethics approval by the Ethics Committee of Gujarat Adani Institute of Medical Sciences. The data in this article are supported by a Medline and Google scholar search using the key words osteoarthritis, osteoarthrosis, temporomandibular joint. These were augmented by references from these articles. The literature and data sources were explored in a thorough and comprehensive manner with the assistance of librarian (PN). This research included studies that examined the management of TMJ osteoarthritis. No limits were applied to the year of study; however I did exclude publications that were not in English and did not have abstracts. Relevant articles were also sought by a hand search review of reference books. All searches were completed by November 2015. Data from the articles were extracted using a predefined data-extraction form. Discrepancies were noted and discussed between the two other faculties (NH and $\mathrm{CR}$ ) in the E.N.T. department and resolved by consensus. Levels of evidence were ascertained for each study. Disagreements were resolved by discussions with 
biostatistician (AP). I evaluated the titles and abstracts of all the studies identified in the initial search to locate any potentially relevant studies. The full texts of studies identified as potentially relevant were then evaluated. Studies were eligible if they included management of TMJ osteoarthritis. The following types of studies were excluded: reviews, commentaries, case reports and letters.

\section{Results}

A combined approach was generally employed in treating patients with TMJ osteoarthritis. Treatment of TMJ osteoarthritis included (Table 1) patients' education and self-management, regular telephone contact, physical therapy, muscle strengthening, appliances and thermal modalities. ${ }^{15,16}$ The evidences for the effectiveness of acupuncture and electrical nerve stimulation were not much. The laser therapy was effective in reducing the pain. The psychological education management improved emotional outcomes. ${ }^{16}$

Table I Studies of Different modalities of treatment of osteoarthritis

\begin{tabular}{|c|c|}
\hline Studies & Treatment Modalities \\
\hline Jamtvedt et al. [16] & Physical therapy \\
\hline Atkins \& Eichler [I7] & Self-massage \\
\hline Inchingolo et al. [20] & $\begin{array}{l}\text { Combined occlusal and pharmacological } \\
\text { therapy }\end{array}$ \\
\hline Manfredini et al. [22] & Injection of Hyaluronic acid \\
\hline Man et al. [24] & Injection of Transforming growth factor-beta \\
\hline Kavas et al. [25] & Raloxifene \\
\hline Wahlund et al. [27] & Occlusal appliance, relaxation training \\
\hline Tanaka et al. [28] & Occlusal stabilization appliance \\
\hline Pandis [29] & $\begin{array}{l}\text { Hard stabilization appliances and non occluding } \\
\text { appliances or no therapy }\end{array}$ \\
\hline McAlindon et al. [34] & Glucosamine and chondroitin \\
\hline Rothacker et al. [35] & $\begin{array}{l}\text { Topical application of } 10 \% \text { trolamine salicylate } \\
\text { cream }\end{array}$ \\
\hline Swięchowicz et al. [36] & Hyaluronic acid intra-articular injections \\
\hline
\end{tabular}

Patients' instruction for the activity and habit modification included soft diet, active and passive jaw movements and relaxation therapies. Patients were advised to avoid gum chewing and excessive mouth opening. ${ }^{10}$ In some patients auto-massaging helped and reduced pain and stiffness of the TMJ. It did not help in improving range of movement. ${ }^{17}$

The TMJ stabilization methods were helpful in redistributing the forces and were also found useful in protecting teeth. They reduced bruxism and joint loading and stabilized occlusion and relaxed masticatory muscles. ${ }^{18}$ In certain patients an anterior repositioning appliance was seen beneficial. ${ }^{19}$ The pharmacotherapy increased benefits of splint therapy. ${ }^{20}$ In highly selective patients with TMJ arthropathies, the intra oral appliances themselves relieved the pain significantly.

Pharmacological treatment included acetaminophen, cyclooxygenase-2 (COX-2), capsaicin, topical and oral non-steroidal anti-inflammatory drugs (NSAIDs), intra-articular steroids and hyaluronidase. NSAIDs reduced pain as well as inflammation and reduction of inflammation slowed down the degeneration. NSAIDs were used with proton pump inhibitors which prevented GI side effects. Although having better GI profile, most of the COX-2 inhibitors were discontinued because of their risk of cerebrovascular accidents. Glucosamine and/or chondroitin sulphate and diacerein were used for their pain relief and structure-modifying effects. Opioid analgesics were used in refractory patients with pain. ${ }^{10,15,21}$
The efficacy of hyaluronic acid (HA) injections was found comparable with corticosteroid injections or oral appliances. ${ }^{22}$ Arthrocentesis combined with HA injections helped in pain and functioning in some patients, however the efficacy of high or low molecular weight HA was equivocal. Intra-articular local anesthetics and corticosteroids (Methylpredinisolone and triamcinoloneacetonide) were suggested when oral medications were not effective. ${ }^{23}$

The other therapies were also tried in the management $t^{24,25}$ such as lubricin, raloxifen, antioxidants, cathepsins, NGF antibodies, strontium ranelate and various growth factors. Because of the heterogeneity in study design, data collection and data presentation, I did not pool summary data in a metaanalysis. There were particular weaknesses in terms of outcome ascertainment and the representative nature of the cases.

\section{Discussion}

The clinical findings of the patient are integrated with preliminary radiographic evidence to make provisional diagnosis which should be confirmed through necessary imaging and laboratory studies for the successful management of the patients with TMJ osteoarthritis.

In diabetic patients topical capsaicin can increase the chances of skin ulcers as it has effects on autonomic nerves. Various guidelines suggest that topical NSAIDs should be tried before prescribing oral NSAIDS..$^{15}$

During early phase (2.5-4years) patient complaints of clicking and locking. Intermediate phase (6months to 1year) is associated with TMJ destruction and patient has arthralgia, trismus, and grating sounds. There is no degenerative activity during the 6months Late phase (burnout phase) and TMJ stabilizes with time. So the non-invasive medical treatment should be preferred over invasive procedures and patients are benefitted with this approach. Some patient may have trismus and grating sounds without pain. ${ }^{11}$

OPG (panoramic view) is the imaging of choice for screening and assessing the TMJ complex. TMJ tomograms are good for osseous condylar abnormalities. Cone beam CT offers a comprehensive view of TMJ bony components as it reproduces images in all the three planes. ${ }^{26}$ The role of oral appliance therapy in the treatment of TMJ osteoarthritis is debatable as certain studies have found resolution of pain with the use of these appliances ${ }^{27-29}$ while others found them ineffective30. The conservative reversible techniques and stabilization procedures offer best results in long run. ${ }^{31,32}$ In the active phase of TMJ osteoarthritis, orthodontic procedures, full mouth therapy and fixed dentures should not be performed.

Acetaminophen (up to $4 \mathrm{~g} /$ day) is preferred and the first line of treatment because of its safety profile. Caution is required in patients who are alcoholics and have liver disorders. However it is not as effective in relieving pain as NSAIDs. Randomized controlled trials report that. The tramadol was found effective and well tolerated analgesic in randomized controlled trials. In refractory patients with pain, opioid analgesics are used. ${ }^{33}$ Among supplements glucosamine and chondroitin are widely used but their effectiveness is equivocal. They are said to have chondro-protective effects. One meta-analysis showed their moderate effectiveness. ${ }^{34}$

The clinical trials of topical diclofenac and ketoprofen reported increased safety and tolerability and found better in controlling pain than placebo. Their effectiveness was seen similar to oral NSAIDs with the exception of piroxicam. But NSAIDs can cause gastrointestinal (GI) problems and needs caution in cases of gastric 
and renal disorders. Topicals trolamine salicylate ${ }^{35}$ was found helpful in some patients. Topical salicylates and capsaicin are not found effective and they can cause unexpected salicylates poisonings and capsaicin-induced nerve desensitization.

Cytokine (glycoproteins) directed therapy (proinflammatory cytokines, TNF $\alpha$ ) have shown an important role in the treatment of osteoarthritis10,23. They curtail cytokines activity and reduce destruction. Early damage to the joint cartilage due to certain metabolic and mechanical factors triggers an immune response and release cytokines and chemokines. There occurs activation of the complement system that leads to release of matrix metalloproteinase (MMPs) and prostaglandin E (PGE) which help in degrading the articular cartilage. These factors eventually lead to remodeling of subchondral bone. ${ }^{10,23}$

TMJ arthrocentesis did not make significant difference with different protocols in outcome. Viscosupplementation, a disease modifying agent is effective in the treatment of osteoarthritis ${ }^{36}$ and helps in pain, inflammation and chondroprotection. Therefore HA injections should be reserved for the patients who are not benefitted with conservative techniques. ${ }^{22}$ Steroids can cause elevated blood sugar, fat atrophy and cartilage softening.

Arthroplasty procedures (condylotomy, disc and joint replacement) are indicated only when conservative measures are not effective. Treatment of TMJ osteoarthritis depends upon the stage of the disease, symptoms and risk factors and is mainly effective in managing inflammation, function, deformity and pain. ${ }^{10}$ The conservative medical treatment is effective in treating these patients ${ }^{31}$ and includes electrical stimulation, physiotherapy, topical ointments, pharmacotherapy, supplements, acupuncture and intra-articular steroid and HA injections. The initial multimodal treatment has shown good results in the long term management. ${ }^{37}$

\section{Conclusion}

The first line of therapy of osteoarthritis of TMJ is noninvasive medical treatment as the advanced stages are not progressive in nature. Diagnosis of different stages of TMJ osteoarthritis is important in deciding the line of treatment. Even the advanced stage is effectively managed by reversible and non-surgical procedures. Conservative modality of therapy is effective and causes least morbidity. Irreversible oral rehabilitation is generally avoided in active stages of TMJ osteoarthritis. It needs careful study to establish the role of supplements and alternative medicine. HA injections should be reserved for the patients who are not benefitted with conservative techniques. Arthroplasty procedures (disc and joint replacement) are indicated only when conservative measures are not effective. After analyzing different techniques of management, it is obvious that the noninvasive conservative line of treatment is effective and should be the cornerstone of therapy for the patients suffering from TMJ osteoarthritis.

\section{Acknowledgements}

The literature and data sources were explored with the assistance of librarian Ms Purvi Nirmal (MLiSc) of Gujarat Adani Institute of Medical Sciences. Discrepancies of data were discussed with Dr. Narendra Hirani (M.S.) and Dr. Chandrasekhar Ranade (M.S.) faculties in the E.N.T. department of Gujarat Adani Institute of Medical Sciences. Levels of evidence and other biostatistics matter were discussed with biostatistician Dr Ajay Pathak (PhD) of P.S.M department of P. S. Medical College.

\section{Funding Sources}

No funding from any source.

\section{Author contributions}

Conceived and designed the study. Analysed the data: Wrote the first draft of the manuscript: Contributed to the writing of the manuscript: Developed the structure and arguments for the paper: Made critical revisions.

\section{Disclosures and ethics}

This article does not contain any studies with human participants or animals performed by any of the authors.

\section{Conflicts of interest}

Author declares there are no conflicts of interest.

\section{References}

1. Bansal M. Diseases of Ear, Nose and Throat. New Delhi. Jaypee Brothers Medical Publishers (P) Ltd., India. 2013. p.668.

2. Jiao K, Niu LN, Wang MQ, et al. Subchondral bone loss following orthodontically induced cartilage degradation in the mandibular condyles of rats. Bone. 2011;48(2):362-371.

3. Dijkgraaf LC, Liem RS, de Bont LG. Ultrastructural characteristics of the synovial membrane in osteoarthritic temporomandibular joints. $J$ Oral Maxillofac Surg. 1997;55(11):1269-1279.

4. Bansal M. Prevalence and diagnostic features of osteoarthrosis of the temporomandibular joint: a review. Int J Res Orthop. 2016;2:1-4.

5. Toller PA. Osteoarthrosis of the mandibular condyle. $\mathrm{Br}$ Dent $\mathrm{J}$. 1973;134(6):223-231.

6. Mejersjo C. Therapeutic and prognostic considerations in TMJ osteoarthrosis: a literature review and a long-term study in 11 subjects. Cranio. 1987;5(1):69-78.

7. Kang SC, Lee DG, Choi JH, , et al. Association between estrogen receptor polymorphism and pain susceptibility in female temporomandibular joint osteoarthritis patients. Int J Oral Maxillofac Surg. 2007;36(5):391-394.

8. Buckwalter JA, Mankin HJ. Articular cartilage: degeneration and osteoarthritis, repair, regeneration, and transplantation. Instr Course Lect. 1998;47:487-504.

9. Buckwalter JA. The role of mechanical forces in the initiation and progression of osteoarthritis. HSS J. 2012;8(1):37-38.

10. Tanaka E, Detamore MS, Mercuri LG. Degenerative disorders of the temporomandibular joint: etiology, diagnosis, and treatment . J Dent Res. 2008;87(4):296-307.

11. Stegenga B, de Bont LG, Boering G, et al. Tissue responses to degenerative changes in the temporomandibular joint: a review. J Oral Maxillofac Surg. 1991;49(10):1079-1088.

12. Chen YJ, Shih TT, Wang JS, et al. Magnetic resonance images of the temporomandibular joints of patients with acquired open bite. Oral Surg Oral Med Oral Pathol Oral Radiol Endod. 2005;99(6):734-742.

13. Schellhas KP, Piper MA, Omlie MR. Facial skeleton remodeling due to temporomandibular joint degeneration: an imaging study of 100 patients. Cranio. 1992;10(3):248-259.

14. Dimitroulis G. The prevalence of osteoarthrosis in cases of advanced internal derangement of the temporomandibular joint: a clinical, surgical and histological study. Int J Oral Maxillofac Surg. 2005;34(4):345-349. 
15. Zhang W, Moskowitz RW, Nuki G, et al. OARSI recommendations for the management of hip and knee osteoarthritis, Part II: OARS evidence-based, expert consensus guidelines. Osteoarthr Cartil. 2008;16(2):137-162.

16. Jamtvedt G, Dahm KT, Christie A, et al. Physical therapy interventions for patients with osteoarthritis of the knee: an overview of systematic reviews. Phys Ther. 2008;88(1):123-136.

17. Atkins DV, Eichler DA. The effects of self-massage on osteoarthritis of the knee: a randomized, controlled trial. Int J Ther Massage Bodywork. 2013;6(1):4-14.

18. Stegenga B, Dijkstra PU, de Bont LG, et al. Temporomandibular jointosteoarthrosis and internal derangement. Part II: Additional treatment options. Int Dent J . 1990;(6):347-353.

19. Madani AS, Mirmortazavi A. Comparison of three treatment options for painful temporomandibular joint clicking. J Oral Sci. 2011;53(3):349-354.

20. Inchingolo F, Tatullo M, Marrelli M, et al. Combined occlusal and pharmacological therapy in the treatment of temporo-mandibular disorders. Eur Rev Med Pharmacol Sci. 2011;15(11):1296-1300.

21. Blumstein H, Gorevic PD. Rheumatologic illnesses: treatment strategies for older adults. Geriatrics. 2005;60(6):28-35.

22. Manfredini D, Piccotti F, Guarda-Nardini L. Hyaluronic acid in the treatment of TMJ disorders: a systematic review of the literature. Cranio. 2010;28(3):166-176.

23. Jacofsky, David J, Anderson, , et al. Osteoarthritis. Hospital Physician. 2005;41(7):17-25.

24. Man C, Zhu S, Zhang B, et al. Protection of articular cartilage from degeneration by injection of transforming growth factor-beta in temporomandibular joint osteoarthritis. OralSurg Oral Med Oral Pathol Oral Radiol Endod. 2009;108(3):335-340.

25. Kavas A, Cagatay ST, Banerjee S, et al. Potential of Raloxifene in reversing osteoarthritis-like alterations in rat chondrocytes: an in vitro model study. J Biosci . 2013;38(1):135-147.

26. Meng JH, Zhang WL, Liu DG,et al. Diagnostic evaluation of the temporomandibular joint osteoarthritis using cone beam computed tomography compared with conventional radiographic technology. Beijing Da Xue Xue Bao. 2017;39(1):26-29.
27. Wahlund K, List T, Larsson B. Treatment of temporomandibular disorders among adolescents: a comparison between occlusal appliance, relaxation training, and brief information. Odontol Scand. 2003; 61(4):203-211.

28. Tanaka EE, Arita ES, Shibayama B. Occlusal stabilization appliance: evaluation of its efficacy in the treatment of temporomandibular disorders. J Appl Oral Sci. 2004;12(3):238-243.

29. Pandis N. Modest improvement in temporomandibular disorder-related pain associated with use of hard stabilization appliances compared with use of non occluding appliances or no therapy. J Am Dent Assoc. 2011;142(11):1295-1296.

30. Nemcovsky CE, Gazit E, Serfati V, et al. Comparative study of three therapeutic modalities in a temporomandibular disorder (TMD) population. Cranio. 1992;10(2):148-155.

31. de Leeuw R, Boering G, Stegenga B, et al. Symptoms of temporomandibular joint osteoarthrosis and internal derangement 30 years after non-surgical treatment. Cranio. 1995;13(2):81-88.

32. de Leeuw R, Boering G, Stegenga B,et al. Radiographic signs of temporomandibular joint osteoarthrosis and internal derangement 30 years after nonsurgical treatment. Oral Surg Oral Med Oral Pathol Oral Radiol Endod. 1995;79(3):382-392.

33. Sinusas K. Osteoarthritis: diagnosis and treatment. Am Fam Physician. 2012;85(1):49-56

34. McAlindon TE, LaValley MP, Gulin JP, et al. Glucosamine and chondroitin for treatment of osteoarthritis: a systematic quality assessment and meta-analysis. JAMA. 2000;283(11):1469-1475.

35. Rothacker DQ, Lee I, Littlejohn TW. Effectiveness of a single topical application of $10 \mid \mathrm{x} \%$ trolamine salicylate cream in the symptomatic treatment of osteoarthritis. J Clin Rheumatol. 1998;4(1):6-12.

36. Swięchowicz S, Ostałowska A, Kasperczyk A, et al. Evaluation of hyaluronic acid intra-articular injections in the treatment of primary and secondary osteoarthritis of the knee. Pol Orthop Traumatol. 2012;22(77):105-109.

37. Langworthy MJ, Saad A, Langworthy NM. Conservative treatment modalities and outcomes for osteoarthritis: the concomitant pyramid of treatment. Phys Sportsmed. 2010;38(2):133-145. 